\title{
Classically conditioned heart rate in rats following preconditioning exposure to the CS
}

\author{
ROBERT D: FITZGERALD and JOHN HOFFMAN \\ Department of Medical Psychology, University of Oregon Health Sciences Center, Portland, Oregon 97201
}

\begin{abstract}
The influence of preexposure to the CS on classically conditioned heart rate was examined in three groups of rats receiving either 0,10 , or $50 \mathrm{CS}$-alone trials prior to the beginning of acquisition training. The conditioned response was a deceleration in heart rate for all groups. Compared to the 50 group, the 0 and 10 groups both showed a lower overall level of conditioning performance and a slower rate of development of the conditioned response. It was suggested that the presence of the nonhabituated orienting response may have interfered with the conditioning process.
\end{abstract}

Preconditioning trials with the CS alone have routinely been used in classical heart-rate conditioning studies in which the direction of the conditioned response and that of the heart-rate orienting response to initial presentations of the CS were the same (Bruner, 1969; Fitzgerald, Martin, \& O'Brien, 1973; Scobie, 1973; Yehle, Dauth, \& Schniederman, 1967). These trials served the purpose of habituating or at least reducing the magnitude of the orienting reaction so that the development of the CR could be observed. That this procedure should perhaps be scrutinized is suggested by the results of recent investigations revealing that preacquisition exposure to the CS can have a deleterious effect on subsequent conditioning performance.

This effect, labeled latent inhibition by Lubow and Moore (1959), has been found in a considerable number of animal studies involving a variety of skeletal responses such as leg flexion (Lubow \& Moore, 1959), tail movement (Chacto \& Lubow, 1967), and eyelid closure (Siegel, 1969). However, in two recent reviews of the literature (Weiss \& Brown, 1974; Lubow, 1973) no studies were cited involving conditioned autonomic responses of animals. The purpose of the present experiment was to determine whether preexposure to the CS adversely affects subsequent heart-rate (HR) conditioning in rats.

\section{METHOD}

\section{Subjects}

Forty-ight hooded Long-Evans female rats, weighing from 220-270 g, were used as subjects. They were purchased from the Animal Care Department at the University of Oregon Health Science Center and housed under continuous illumination in small colony cages with food and water available ad lib.

This research was supported by National Science Foundation Grant BMS74-18542, by an Oregon Heart Association Grant, in part by a U. S. Public Health Service Grant, 5 S01 RR05412, and National Institute of General Medical Science Grant 5 T01 GM01495.

Requests for reprints should be sent to Robert D. Fitzgerald, Department of Medical Psychology, University of Oregon Health Sciences Center, Portland, Oregon 97201.

\section{Apparatus}

Subjects were physically immobilized in a U-shaped Plexiglas restrainer manufactured by $\mathrm{E} \& \mathrm{M}$ Instrument Company Inc. The restrainer was located in an Industrial Acoustic sound isolation chamber equipped with an exhaust fan. To further reduce the possibility of extraneous auditory signals reaching the rat, white noise measuring $75 \mathrm{~dB}$ was continuously presented in the chamber. Two rats were conditioned concurrently with trials alternating between the rats. The electrocardiogram (ECG) was recorded on a Grass polygraph from 20-ga hypodermic needles inserted under the skin on either side of the rat's thoracic cavity. Heart beats occurring in consecutive time intervals within each trial were automatically tabulated by means of an on-line system consisting of a low-force lever-type microswitch mounted directly above the ECG polygraph pen and adjusted so that the $R$ wave of the QRS complex actuated the switch (Fitzgerald, Vardaris, \& Teyler, 1968). The heart beat totals were punched out on a Tally high speed paper-tape perforator and the tapes were processed on a Wang Model $720 \mathrm{C}$ minicomputer.

The CS was a $6.3-\sec 85 \mathrm{~dB} 2.9 \mathrm{kHz}$ tone produced by a 1 -in. Mallory sonar alert mounted directly in front of the subject. The US was a .3-sec 1.4 mA 60-cycle ac electric shock delivered to the base of the rat's tail through No. 6 machine screws that were held in place approximately $2-3 \mathrm{~cm}$ apart with small pieces of elastic rubber tubing. The CS-US interval was $6 \mathrm{sec}$ with the US overlapping the final $.3 \mathrm{sec}$ of the CS. Trials were initiated automatically by a film-tape programmer with the stimulus events occurring within a trial controlled by transistorized logic modules.

Procedure

The basic experiment consisted of three experimental groups and one control group of 12 rats each. Each experimental group received either 0,10 , or 50 preconditioning trials with CS alone at intertrial intervals (ITI) of 70,90 , or $110 \mathrm{sec}$ (mean $90 \mathrm{sec}$ ) followed by 30 paired conditioning trials at ITIs of 150,180 , or $200 \mathrm{sec}$ (mean $180 \mathrm{sec}$ ). The control group was composed of three subgroups of four rats each receiving either 0,10 , or 50 CS-alone trials followed by 30 unpaired presentations of the CS and US with the CS occurring 70,90, or 110 sec (mean $90 \mathrm{sec}$ ) after the US. The first CS-alone trial in the $50 \mathrm{CS}$-alone condition was given $15 \mathrm{~min}$ after the rats were placed in the restrainer. The series of $10 \mathrm{CS}$-alone trials began $90 \mathrm{~min}$ after the rats were restrained. Rats receiving $0 \mathrm{CS}$-alone trials were given their first conditioning trial $106 \mathrm{~min}$ after being restrained. These times were selected so that the duration of restraint prior to the beginning of conditioning would be the same for all subjects.

The change in HR elicited by the CS on the CS-alone and conditioning trials was obtained for each rat by subtracting the 


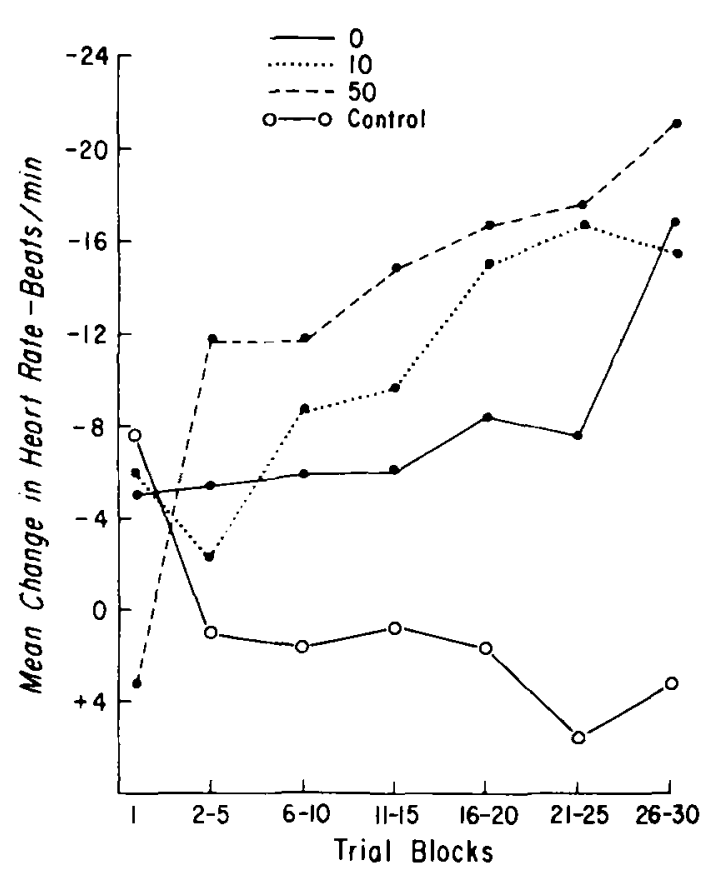

Figure 1. Mean CS minus pre-CS heart-rate changes of the experimental and control groups as a function of trials during acquisition.

number of heart beats during the 6 sec preceding the onset of the CS (pre-CS period) from the number during the 6-sec CS-US interval. These difference score values were then converted to beats per minute $(\mathrm{bpm})$.

\section{RESULTS}

An analysis of variance of pre-CS HR in five-trial blocks of acquisition showed no significant differences between the groups. The orienting response to the CS of the 50 group changed reliably from a deceleration of $5.0 \mathrm{bpm}$ on the first five CS-alone trials to an acceleration of $4.0 \mathrm{bpm}$ on the last five trials $(F=8.05$, $\mathrm{df}=1 / 11, \mathrm{p}<.05)$. The magnitude of the decelerative orienting response of the 10 group decreased from 4.0 bpm on the first five trials to $1.0 \mathrm{bpm}$ on the last five trials but this change was not significant.

In order to obtain a measure of the preconditioning HR change to the CS in the 10 and 50 groups which would be equivalent to that available for the 0 group, the first acquisition trial was selected for all groups. Figure 1 shows the CS minus pre-CS scores of the experimental and control groups averaged over the first trial, over the next four trials, and then over successive five-trial blocks of acquisition. Since the three control groups did not differ significantly from each other their data were combined to form a single group. Inspection of Figure 1 reveals that the HR responses of the 0 and 10 experimental groups on the first conditioning trial were decelerations whereas for the 50 group it was an acceleration $(t=1.75, \mathrm{df}=34, \mathrm{p}<.05)$. On subsequent trials the accelerative response of the 50 group changed rapidly to a decelerative reaction whose magnitude was greater throughout acquisition than that shown by either the 0 or 10 group. In fact, the 0 group showed little evidence of conditioning during the first 25 trials of acquisition. An analysis of variance in which the HR response on the first conditioning trial was subtracted from each of the remaining trial blocks for the experimental and control groups produced a significant effect of groups $(F=6.43$, df $=3 / 44, p<.01)$, a significant effect of trials $(F=6.48, \mathrm{df}=5 / 220, \mathrm{p}<.01)$, and a significant Groups by Trials interaction $(F=2.19$, $\mathrm{df}=15 / 220, \mathrm{p}<.001)$. A Newman-Keuls test revealed that each of the experimental groups was significantly different from the control group and that the 50 experimental group was significantly different from the 0 and 10 experimental groups $(\mathrm{p}<.01)$. The 0 and 10 experimental groups did not differ reliably from each other. The same analyses were carried out using the HR responses of the 10 and 50 groups on the last five CS-alone trials as a correction factor. These tests provided essentially the same outcomes with the addition that the 0 and 10 groups were now significantly different from each other.

\section{DISCUSSION}

The present experiment provided no evidence of the latent inhibition phenomenon frequently obtained in classical conditioning studies involving skeletal responses. On the contrary, the group receiving 50 preconditioning trials with the CS alone showed a higher overall CR level and a faster rate of development of the $\mathrm{CR}$ than the groups receiving either 0 or 10 pretest trials. Growth in CR magnitude of the 0 group appeared to be especially retarded until the last five acquisition trials, at which point the $\mathrm{CR}$ increased abruptly to approximately the same level as that achieved by the other two groups. One methodological implication of these findings is that failure to give preacquisition trials with the CS alone can have an adverse effect on subsequent HR conditioning in animals. The required number of such trials to maximize conditioning could and probably does vary with the species being examined.

In general, the present results agree with those reported by Silver (1974) who found that magnitude of a conditioned skin-conductance response in humans was increased by preconditioning exposure trials to the $\mathrm{CS}$, with the number of such trials given being either 1 , 4 , or 16 . That a larger number of CS-alone trials may depress conditioning of the electrodermal response in humans was indicated in a study by Surwit and Poser (1974). They reported that the probability of occurrence of short-latency changes in electrodermal activity was reduced throughout conditioning by giving 50 or 100 preconditioning trials with the CS. During early conditioning trials long latency responses also provided 
evidence of latent inhibition. However, consonant with the results obtained in the Silver (1974) experiment, magnitudes of electrodermal responses were not influenced by pretest CS-alone trials.

The orienting response elicited by a novel stimulus has generally been viewed as a complex reaction of the total organism involving autonomic, skeletal-motor and cortical components (Sokolov, 1963). Pavlov (1927) recognized the importance of the orienting response in his treatment of classical conditioning, noting that the presence of a vigorous reaction to the CS at the beginning of conditioning can seriously impair development of the CR. A similar type of interference may have been responsible for the relatively poor conditioning performance shown by the 0 and 10 groups in the current experiment. However, it seems unlikely that this would be produced by the HR component of the orienting response, as this reaction was quite small in both groups. On the other hand, the fact that the orienting response and the conditioned response were in the same direction may have increased the likelihood of competition between the two reactions. It may also be relevant to note in this connection that one feature shared by many of the traditional classical conditioning studies demonstrating latent inhibition is that they have involved skeletal reactions that are not specifically elicited by a CS prior to conditioning.

In several recent studies (e.g., Fitzgerald \& Martin, 1971; Fitzgerald \& Teyler, 1970), it was suggested that the HR CR in rats may be a modified version of the HR orienting response. According to this hypothesis, the conditioning process is initiated by the US activating the neural circuits mediating the orienting response. Once it occurs, the CR is then modified by parameters such as the CS-US interval and the intensity of the US. Support for the position of a linkage between the CR and orienting response has been provided by studies showing (1) that the direction of both responses was the same (Fitzgerald \& Martin, 1971; Fitzgerald \& Teyler, 1970), (2) that both responses were controlled primarily by increased vagal output (Fitzgerald, Martin, \& O'Brien, 1973), and (3) that the detailed topography of the orienting reaction, which was modified during conditioning, tended to return during extinction (Fitzgerald \& Teyler, 1970). However, the fact that CR performance of the 0 group in the current experiment was not facilitated by the presence of the nonhabituated orienting response is seemingly inconsistent with this hypothesis. While admittedly speculative, one possible explanation is that the proposed energizing effects of the US on the vagal network mediating the orienting response were attenuated in the 0 group by ongoing activity in that network. In the case of the 50 group, the CS-alone trials leading as they did to HR acceleration, may have reduced the amount of this activity, allowing the US to have a more immediate effect.

\section{REFERENCES}

Bruner, A. Reinforcement strength in classical conditioning of leg flexion. freezing and heart rate in cats. Conditional Reflex, $1969,4,1,24-31$.

Chacto, C., \& Lubow, R. E. Classical conditioning and latent inhibition in the white rat. Psychonomic Science, 1967, 9, 135-136.

Fitzgerald, R. D., \& Martin, G. K. Heart-rate conditioning in rats as a function of interstimulus interval. Psychological Reports, 1971, 29, 1103-1110.

Fitzgerald, R. D., \& Teyler, T. J. Trace and delayed heart rate conditioning in rats as a function of US intensity. Journal of Comparative and Physiological Psychology, 1970, 10. 242-253.

Fitzgerald, R. D., Martin, G. K. \& O'Brien, J. H. Influence of vagal activity on classically conditioned heart rate in rats. Journal of Comparative and Physiological Psychology, 1973, 83, 485-491.

Fitzgerald, R. D., Vardaris, R. M., \& Teyler, T. J. An on-line method for measuring heart rates in conditioning experiments. Psychophysiology, 1968, 4, 352-353.

Lubow, $R$. E. Latent inhibition. Psychological Bulletin, 1973, $79,398-407$.

Lubow, R. E., \& Moore, A. U. Latent inhibition: The effect of nonreinforced preexposure to the conditioned stimulus. Journal of Comparative and Physiological Psychology, 1959, $52,416-419$.

Pavlov, I. P. Conditioned reflexes. (G. V. Anrep, Ed. and trans.) New York: Dover, 1927.

Scobie, $S$. $R$. Unconditional stimulus intensity and cardiac conditioning in the goldfish. Physiology and Behavior, 1973, 11, 31-34.

Siegel, S. Effects of CS habituation on eyelid conditioning. Journal of Comparative and Physiological Psychology, 1969, 68, 245-248.

Silver, A. I. Effects of prior CS presentations on classical conditioning of the skin conductance response. Psychophysiology, $1973,10,583-588$.

Sokolov, E. N. Higher nervous functions: The orienting reflex. Annual Review of Psychology, 1963, 25, 545-580.

Surwit, R. S., \& Poser, E. G. Latent inhibition in the conditioned electrodermal response. Journal of Comparative and Physiological Psychology, 1974, 86, 543-548.

Weiss, K. R., \& Brown, B. L. Latent inhibition: $A$ review and a new hypothesis. Acta Neurobiologica Experimentalis, 1974, 34, 301-316.

Yehle, A., Dauth, G., \& Schneiderman, N. Correlates of heart rate classical conditioning in curarized rabbits. Journal of Comparative and Physiological Psychology, 1967, 64, 98-104.
(Received for publication March 28, 1975. Revision accepted July 21, 1975.) 